\title{
Simulations of the structure and dynamics of nanoparticle-based ionic liquids $\dagger$
}

\author{
Bingbing Hong, Alexandros Chremos \\ and Athanassios Z. Panagiotopoulos*
}

\author{
Received 20th April 2011, Accepted 19th May 2011 \\ DOI: 10.1039/c1fd00076d
}

\begin{abstract}
We use molecular dynamics simulations over microsecond time scales to study the structure and dynamics of coarse-grained models for nanoparticle-based ionic liquids. The systems of interest consist of particles with charged surface groups and linear or three-arm counterions, which also act as the solvent. A comparable uncharged model of nanoparticles with tethered chains is also studied. The pair correlation functions display a rich structure resulting from the packing of cores and chains, as well as electrostatic effects. Even though electrostatic interactions between oppositely charged ions at contact are much greater than the thermal energy, we find that chain dynamics at intermediate time scales are dominated by chain hopping between core particles. The uncharged core particles with tethered chains diffuse faster than the ionic core particles.
\end{abstract}

\section{Introduction}

Nanoscale ionic materials (NIMs) consist of inorganic core particles surface-functionalized with ionic groups and dispersed in a medium consisting of large organic counterions, which also act as the solvent. ${ }^{1-6}$ With appropriate choice of the counterion groups they exist as liquids at room temperature. ${ }^{7}$ They share with conventional ionic liquids the desirable properties of having a hybrid chemical character and negligible vapor pressures. ${ }^{8}$ Their physicochemical characteristics can be tuned by varying the core nanoparticle composition and size, as well as the chemical character and molecular weight of the counterions..$^{9-11}$ A related class of materials, nanoscale organic hybrids ${ }^{2,12-14}$ (NOHMs), share many chemical and structural characteristics with NIMs and can also exist as solvent-free liquids. Tethering of traditional ionic liquid constituent ions with nanoparticles has also been proposed.

The synthesis approach for NIMs has evolved over time. First-generation NIMs were made with cationic organosilanes grafted to the nanoparticle cores, with anionic organic compounds as counterions. Second-generation NIMs synthesis entails sulfonic-acid terminated organosilanes grafted to the nanoparticles, with primary or tertiary amine-functionalized oligomers as counterions. Typical second-generation NIMs with tertiary amine groups are shown schematically on the left side of Fig. 1. Each of the oligomer chains on the amines typically consists of a few dozen methylene or ethoxy groups. Finally, third-generation NIMs are made by taking advantage of inherent surface acidic groups (silanol) or grafted (fullerol) hydroxyl groups neutralized with amine-functionalized linear oligomers. A

Department of Chemical and Biological Engineering and Institute for the Science and Technology of Materials, Princeton, NJ 08544, USA. E-mail: azp@princeton.edu

$\dagger$ Electronic supplementary information (ESI) available: Movies showing the microscopic dynamics of chain, ion and core particle motions. See DOI: 10.1039/c1fd00076d 

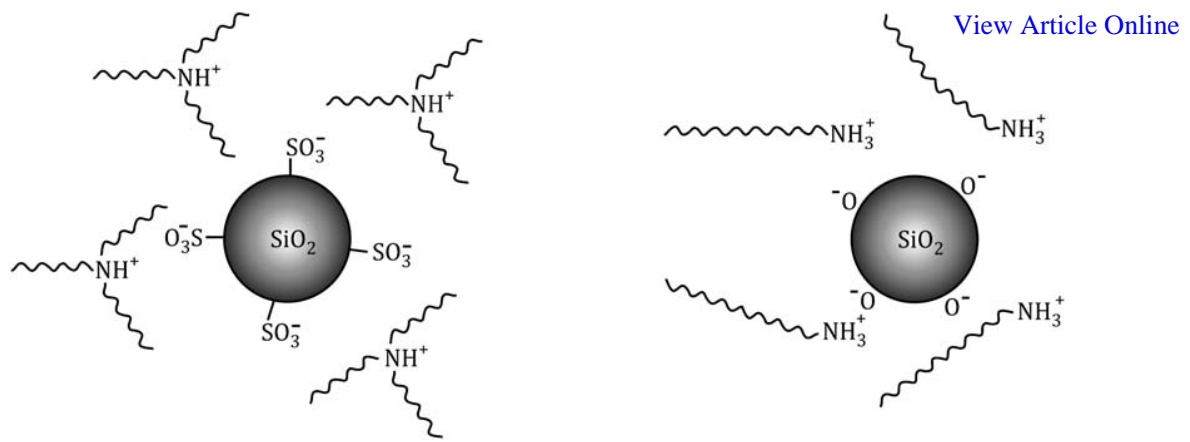

Fig. 1 Left: second-generation NIMs with tertiary amine; right: third-generation NIMs with primary amine.

schematic representation of third-generation NIMs with primary amine counterions is shown on the right side of Fig. 1.

The structure and dynamics of NIMs have been investigated experimentally by techniques that include light scattering, dielectric spectroscopy, rheology, and NMR. ${ }^{7,15,16}$ Water's high relative permittivity lowers effective interactions in ionic solutions, but water is not present in significant amounts in NIMs systems. Thus, oppositely charged ions are expected to interact strongly, with energies many times greater than the thermal energy. The structure and electrical conductivity of NIMs are clearly influenced by the mobility of the ionic groups. However, the reasons for their existence as liquids are not completely understood. ${ }^{7}$ One of the objectives of the present work is to provide a microscopic picture of charged group dynamics in NIMs and possible explanations for their fluidity. Even though simulation studies of conventional ionic liquids (e.g. ref. 17-19) and polymer nanocomposites (e.g. ref. 12, 20-22) are available, simulations of NOHMs and NIMs have not been previously attempted, to the best of our knowledge. In a recent study, ${ }^{23} \mathrm{NOHMs}$ structural features were obtained by field-theoretic methods.

The structure of this paper is as follows. In section 2, we define the three model systems studied and quantify the parameters for the interactions taken into account in the models. Simulation methods and technical details are described in section 3 . In section 4.1, structural characteristics of the model systems are analyzed through the behavior of the pair correlation functions. Section 4.2 deals with the dynamics of diffusion of nanoparticle cores and motion of chains on the surface of a core and from core to core. The paper closes with overall conclusions and possible directions for future work.

\section{Models}

Three different models were studied, to provide qualitative representations of NIMs with primary and tertiary amines, as well as NOHMs. The NOHMs model system is the simplest and forms the basis for the two NIMs models. It is shown schematically in Fig. 2; all computations were performed in three dimensions, but a two-dimensional representation of the model is shown for clarity.

The NOHMs model is comprised of spherical structureless nanoparticles with attached linear oligomer chains. Each chain consists of 15 beads connected via harmonic springs, but otherwise free of bond bending or torsional constraints. The overall length of the chains is within the range of experimentally relevant "canopy" oligomers used in NIMs synthesis. ${ }^{10}$

Interactions between oligomer beads are described by a cut-and-shifted LennardJones potential with cutoff distance $r_{\mathrm{c}}=2.5 \sigma$ : 


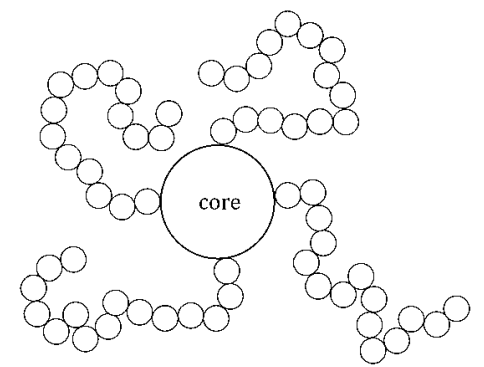

Fig. 2 Schematic illustration of the (three dimensional) NOHMs model nanoparticle.

$$
V(r)=\left\{\begin{array}{cc}
4 \varepsilon\left[\left(\frac{\sigma}{r}\right)^{12}-\left(\frac{\sigma}{r}\right)^{6}\right]-V_{\mathrm{c}} & r \leq r_{\mathrm{c}} \\
0 & r>r_{\mathrm{c}}
\end{array}\right.
$$

where $V_{\mathrm{c}}$ is the value of the (unshifted) Lennard-Jones potential at $r=r_{\mathrm{c}}$.

The size and energy parameters of the chain beads were obtained so as to represent approximately an ethoxy repeat unit $\left(-\mathrm{CH}_{2} \mathrm{OCH}_{2}-\right)$, as follows. Since the ethoxy repeat unit does not exist as a free molecule, the group contribution method of Constantinou and $\mathrm{Gani}^{24}$ was used to obtain an estimated critical temperature of $T_{\mathrm{C}}=409$ $\mathrm{K}$ and critical volume, $V_{\mathrm{C}}=0.124 \mathrm{~L} \mathrm{~mol}^{-1}$. These critical parameters are close to the experimentally measured values for dimethyl ether, $\mathrm{CH}_{3} \mathrm{OCH}_{3}$. The reduced critical parameters of the cut-and-shifted Lennard-Jones fluid with $r_{\mathrm{C}}=2.5 \sigma$ have been obtained by $\mathrm{Smit}^{25}$ as $k_{\mathrm{B}} T_{\mathrm{C}} / \varepsilon=1.085$ and $\sigma^{3} / V_{\mathrm{C}}=0.317$, where $k_{\mathrm{B}}$ is Boltzmann's constant. Thus, the energy parameter for the bead-bead interactions was obtained as $\varepsilon / k_{\mathrm{B}}=377 \mathrm{~K}$, and the size parameter was obtained as $\sigma=0.40 \mathrm{~nm}$. The bead mass was set to $m_{\mathrm{b}}=44 \mathrm{~g} \mathrm{~mol}^{-1}$, appropriate for the ethoxy group.

The harmonic bond potential acting between adjacent beads is:

$$
V_{\mathrm{H}}(r)=k(r-\sigma)^{2}
$$

The spring constant was set to $k=1000 \varepsilon / \sigma^{2}$, which is sufficiently stiff that bond lengths remain within $10 \%$ of their equilibrium values.

The nanoparticle core diameter was set to $d=5 \sigma(=2.0 \mathrm{~nm})$, in the low range of values relevant for the experimental systems. ${ }^{6,7}$ The mass of each core particle was set to $m_{\mathrm{c}}=(d / \sigma)^{3} m_{\mathrm{b}}$, corresponding to a mass density for the particles of $2.2 \mathrm{~g}$ $\mathrm{cm}^{-3}$, appropriate for $\mathrm{SiO}_{2}$. For the core-core and core-bead interactions, we used the purely repulsive Weeks-Chandler-Andersen (WCA) potential, ${ }^{26}$ modified to take into account the differences in particle size, ${ }^{27}$

$$
V_{W C A}(r)=\left\{\begin{array}{cc}
4 \varepsilon\left[\left(\frac{\sigma}{r-\Delta_{i j}}\right)^{12}-\left(\frac{\sigma}{r-\Delta_{i j}}\right)^{6}+\frac{1}{4}\right] & r \leq r_{\mathrm{m}} \\
0 & r>r_{\mathrm{m}}
\end{array}\right.
$$

where $r_{\mathrm{m}}=2^{1 / 6} \sigma+\Delta_{i j}$. The same values of parameters $\varepsilon$ and $\sigma$ were used for the corecore and core-bead interactions as for the bead-bead interactions. The shift distance was set to $\Delta_{\mathrm{cc}}=d-\sigma$ for the core-core interactions, and $\Delta_{\mathrm{cb}}=(d-\sigma) / 2$ for the corebead interactions. The potentials as a function of distance are shown in Fig. 3.

Each nanoparticle has 25 grafted oligomers, so that the grafting density is 2.0 chains $\mathrm{nm}^{-2}$, within the range of experimentally achievable values. ${ }^{2,9}$ The attachment points were fixed on the surface of the core particles for the production periods of the 


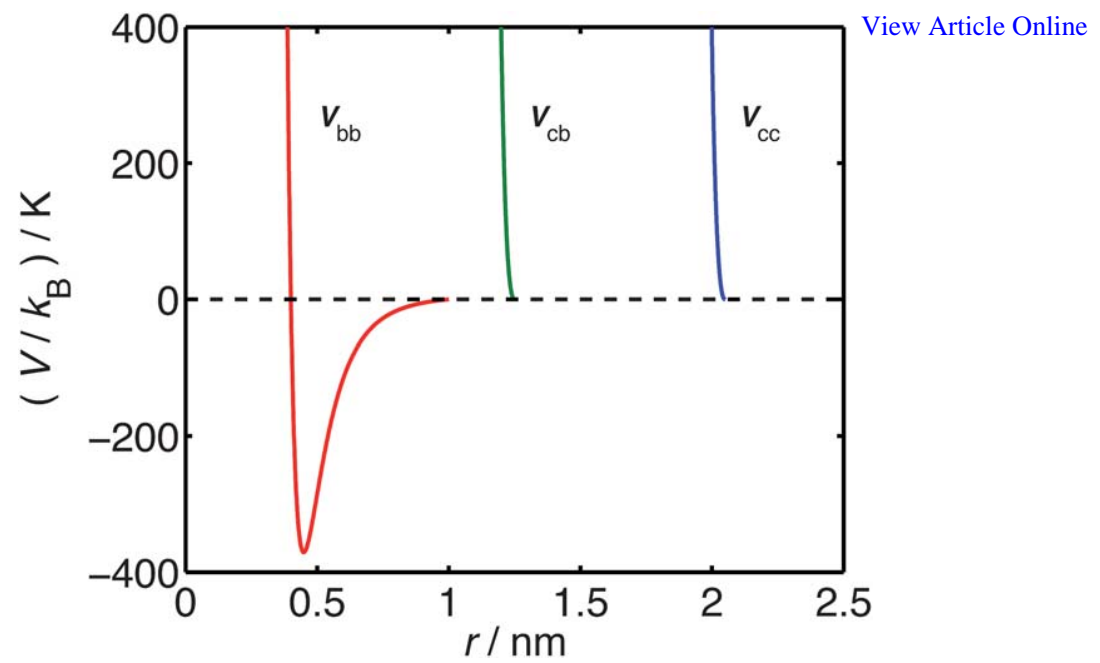

Fig. 3 Potentials acting between beads $\left(V_{\mathrm{bb}}\right)$, between cores and beads $\left(V_{\mathrm{cb}}\right)$, and between cores $\left(V_{\mathrm{cc}}\right)$, as functions of distance.

simulations, so that the core particles move as rigid bodies together with the first beads of the chains. This was done to represent covalently grafted chemical groups. The attachment positions were obtained from initial simulations in which a random distribution of attachment points on the surface of the particles was allowed to evolve, subject to forces from a harmonic spring of the same constant as in eqn (2) and WCA repulsions of range $2.1 \sigma(=0.84 \mathrm{~nm})$ between surface beads. This was done in order to avoid steric overlaps between chains near their attachment points.

The NIMs model with primary amines (termed "NIMs-L" for "linear" from here on) is identical to the model of NOHMs, except that the first bead on the surface of the cores is given a negative charge of $-e\left(=-1.6 \times 10^{-19} \mathrm{C}\right)$, and the bond between the first and second bead of the chain is severed so that the chains, now consisting of 14 beads, are no longer covalently attached to the nanoparticles. The surface sites are immobile, which is appropriate for "third-generation" NIMs systems. A charge of $+e$ is assigned to one of the two terminal beads of the chain to represent the ammonium group.

The NIMs model with tertiary amines is similar, except that the bond between second and third beads of the NOHMs model is now severed and the second bead given a negative charge. This results in a negative site with more freedom to move, appropriate for "second-generation" NIMs systems. The topology of the free chains is also changed to that of a three-armed star with four-bead chains attached to a central bead of charge of $+e$, to represent the tertiary ammonium group. This model is termed "NIMs-S" for "star" from this point on. The NIMs-L and NIMs-S models are shown schematically in Fig. 4.

Electrostatic interactions between charged groups $i$ and $j$ in the NIMs models are given by:

$$
V_{\mathrm{E}}(r)=\frac{1}{4 \pi \varepsilon_{0} \kappa} \frac{q_{i} q_{j}}{r}
$$

where $q$ is the charge of the corresponding group, $\varepsilon_{0}$ the dielectric permittivity of vacuum $\left(=8.85 \times 10^{-12} \mathrm{~F} / \mathrm{m}\right)$ and $\kappa$ is the relative permittivity of the medium, necessary because our coarse-grained models contain structureless core particles and do not take into account the polar character of the ethoxy groups. Polyethylene oxide has a relative permittivity that decreases with molecular weight, with $\kappa=13.7$ for molecular weight of 600 , from ref. 28 . Silica and many non-conjugated polymers have relative permittivities near $\kappa=4,{ }^{29}$ which we adopt in this study. This value 


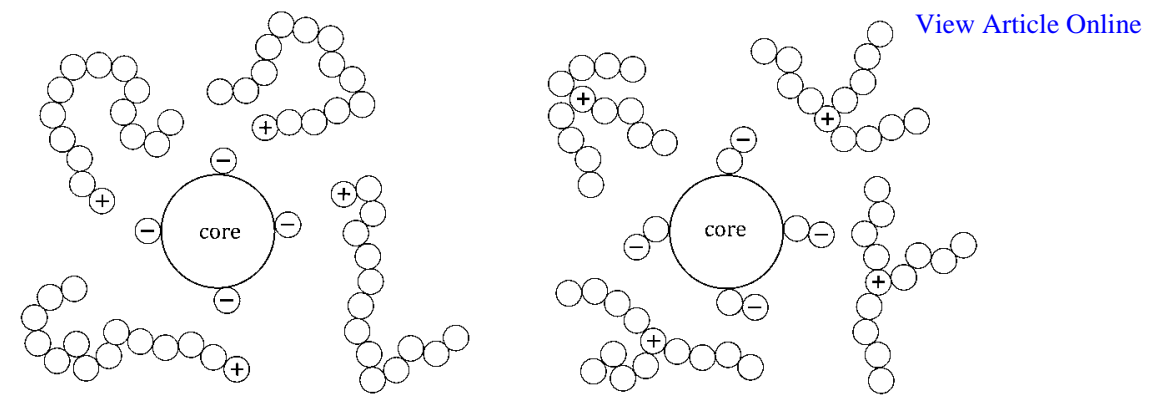

Fig. 4 Schematic of the (three-dimensional) NIMs models: left: NIMs-L; right: NIMs-S.

of $\kappa$ results in electrostatic energy of two oppositely charged beads at contact, $V_{\mathrm{E}}(\sigma)$ $=35 k_{\mathrm{B}} T$ at the temperature of the calculations $(T=300 \mathrm{~K})$.

\section{Methods}

The LAMMPS code ${ }^{30}$ available from http://lammps.sandia.gov, was used for the calculations. Electrostatic summations were performed with the particle-particle particle-mesh method with grid size of $72 \times 72 \times 72$, resulting in each charge spanning 5 grid cells in each spatial dimension. The relative accuracy of the electrostatic energy calculation with these parameters was $10^{-4}$. After initial trials to determine the maximum allowable time step consistent with energy conservation and longterm stability of the simulations, the reduced time step was set to $\delta t^{*}=0.008$. This corresponds to a real time step of $\delta t=\delta t^{*} \sigma \sqrt{m_{\mathrm{b}} / \varepsilon}=12$ fs. Energy was conserved to within $0.05 \%$ for the NIMs and within $0.08 \%$ for the NOHMs "production" simulation runs.

The base system size studied consisted of $N=100$ nanoparticles, or 37600 total interaction centers, of which 5000 were charged in the NIMs models. The box length was determined from a series of constant-temperature simulations at $T=300 \mathrm{~K}$, so as to obtain near-zero pressure (corresponding to atmospheric conditions) for the NOHMs model system. This resulted in a box length of $L=14.8 \mathrm{~nm}$, corresponding to an overall mass density of $1.1 \mathrm{~g} \mathrm{~cm}^{-3}$. The volume fraction of core nanoparticles in the system, defined as the sum of volumes of the core particles divided by the total system volume, is $13 \%$. The systems were initialized in the following way. The nanoparticles were first placed randomly in a cubic simulation box of size $L=220 \mathrm{~nm}$ so that no two particles were closer than $7 \mathrm{~nm}$. The chains on each particle were then randomly grown such that there was no overlap between them and the particles. The simulation box was subsequently reduced at a rate of $0.001 \mathrm{~nm}$ per time step until the desired box length was reached. After the initial equilibration at constant temperature, the production simulations were performed in the $N V E$ ensemble, to avoid any spurious effects of a thermostat on the dynamics. The kinetic temperature of the systems during the production runs averaged $306 \mathrm{~K}$. A smaller system with $N=$ 50 nanoparticles $(L=11.7 \mathrm{~nm})$ was also studied to investigate finite-size effects.

Simulations were performed on multiple Intel X5650 2.66 GHz 6-core processors connected by Infiniband switches. For the base system size of $N=100$ nanoparticles with time step $\delta t^{*}=0.008$, runs on 36 processor cores produced approximately $24 \mathrm{~ns}$ of simulation data per wall-clock day for NIMs. Runs for NOHMs were significantly faster, given the absence of electrostatic interactions; they produced $120 \mathrm{~ns}$ of simulation data per wall-clock day on 24 processor cores.

The mean-squared displacement and ion association correlation functions were determined from snapshots separated by 1000 time steps (12 ps). Separate origins corresponding to the initial $25 \%$ of a run were used, thus allowing correlation 
functions to be computed for times up to $75 \%$ of the total run time. We define assore Online ciation between positive ions and negative ions by a distance criterion with cutoff distance of $0.62 \mathrm{~nm}$. This distance corresponds to the first minimum in the positive-negative pair correlation function. If multiple centers satisfy the distance criterion, the positive ion is considered associated with the center of lowest index. At any given time, we count how many of the ions that were originally within the association radius of a surface site or core particle still satisfy the distance criterion, irrespective of their trajectories at intermediate times.

\section{Results and discussion}

\subsection{Structure}

The pair correlation functions between core particles are shown in Fig. 5. For the NOHMs systems, the core-core correlation function, $g_{\mathrm{cc}}$, has a broad first peak with fine structure, and a weaker second peak. The NIMs-L system has a sharp first peak at a distance of $2.4 \mathrm{~nm}$, corresponding to a single bead separating two core particles. Since negative surface sites are rigidly attached to the core particles, this means that negative surface layers of the two core particles interpenetrate. This is energetically favored because of the presence of positive ions "bridging" nearby cores by interacting strongly with negatively charged surface sites. As will be discussed in Section 4.2, this structural feature has important implications on dynamics, allowing for chains to "hop" from core to core, and providing a path for conductivity. A strong "correlation hole" is present for the NIMs-L system around distances of $3 \mathrm{~nm}$. The NIMs-S system has a structure for $g_{\mathrm{cc}}$ similar to that of NOHMs, but with a slightly higher first peak with no fine structure, deeper trough near $r=4.8 \mathrm{~nm}$, and slightly higher broad second peak. The negative ions are attached to a bead, rather than the surface of the core particles in the NIMs-S model, so they tend to be further away from their cores. Also, because of steric hindrances of the tertiary amines in the NIMs-S model, they are present in much lower amounts on the core surfaces relative to the linear chains of the NIMs-L model. The first peak of the NIMs-S $g_{\mathrm{cc}}$ rises from zero at a larger distance than NOHMs, indicating that the cores are, on average, further apart from each other relative to the NOHMs or NIMs-L systems.

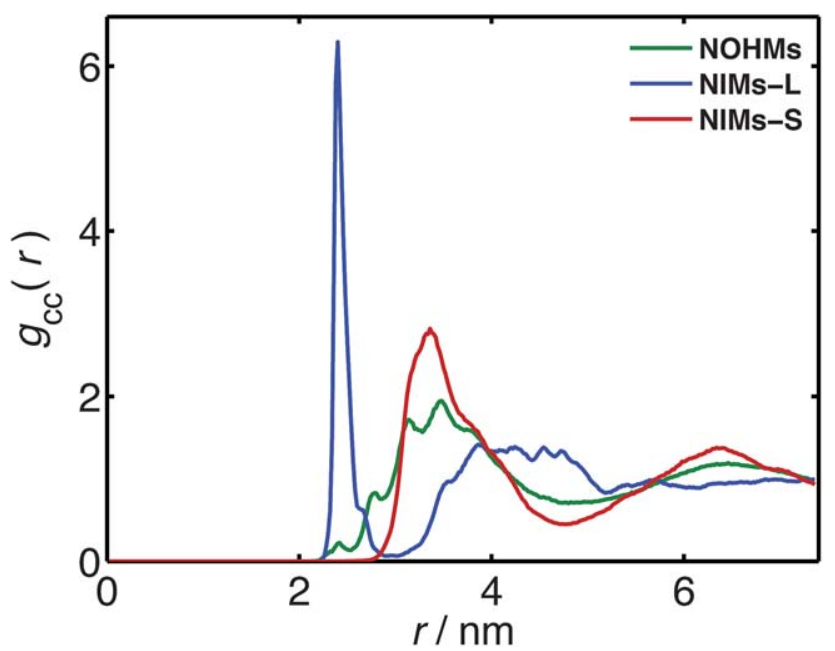

Fig. 5 Pair correlation functions between core particles for the base system $(N=100)$. Green: NOHMs; blue: NIMs-L; red: NIMs-S. 
Fine ripples are present in the NOHMs $g_{\text {cc }}$, with period equal to $\sigma(0.4$ vina $)$, Astigfe Online gesting subtle packing of beads around the cores. However, these ripples are absent in the NIMs-S system and much attenuated in the NIMs-L system. Their presence in NOHMs is a consequence of the tethered character of the chains. For NIMs-L, the positively charged beads at the end of the linear chains bridge two core particles, resulting in a strong first peak, as is also discussed in connection to the core-bead correlation functions. These strongly held chains have a partially tethered character and lead to a slight modulation of $g_{\text {cc }}$.

The pair correlation functions, $g_{\mathrm{cb}}$, between core particles and the second beads of the chains are shown in Fig. 6. All three models display two strong main peaks, the first corresponding to the bead directly on the surface of the core particle (at $r=1.2 \mathrm{~nm}$ ), and the second corresponding to one intervening bead (at $r=1.6 \mathrm{~nm}$ ). Another common feature of these core-bead correlation functions is the existence of a "correlation hole" from 1.6 to $2 \mathrm{~nm}$. However, the heights of the two first peaks are quite different for the three model systems. For NIMs-L, for which the second bead is positively charged and strongly attracted to the negative sites on the cores, the first peak is an order of magnitude higher than the second peak. The linear (primary) amines can penetrate near the core surface, allowing their charged ends to interact with the surface groups. For NOHMs, with an uncharged second bead, the first peak is about twice as high as the second one; both peaks are due to bonded interactions combined with packing effects. For NIMs-S, for which the second bead is negatively charged, the second peak is higher than the first, because the beads prefer to be in contact with counterions (tertiary amine groups) that cannot penetrate as easily as linear chains near the surface of the cores. Another reason for the higher second peak in the NIMs-S system is the mutual repulsion of negatively charged groups tethered to the cores.

Fig. 7 shows the pair correlation functions between core particles and the seventh bead of the chains. For NIMs-S, this is the central, positively charged bead, while for NOHMs and NIMs-L the seventh bead is uncharged. The first two peaks for NIMs$\mathrm{S}$, located at $1.3 \mathrm{~nm}$ and $1.5 \mathrm{~nm}$, have almost the same positions as the first two peaks of core-second bead $g(r)$ shown in Fig. 6. This indicates that although they are subject to steric hindrance, the charged beads of the star chains are still able to get near the cores; however, the peak height is only around 3 for the tertiary amines, whereas the linear chains had a peak height of more than 20. The structure of $g_{\mathrm{cb}}$ is

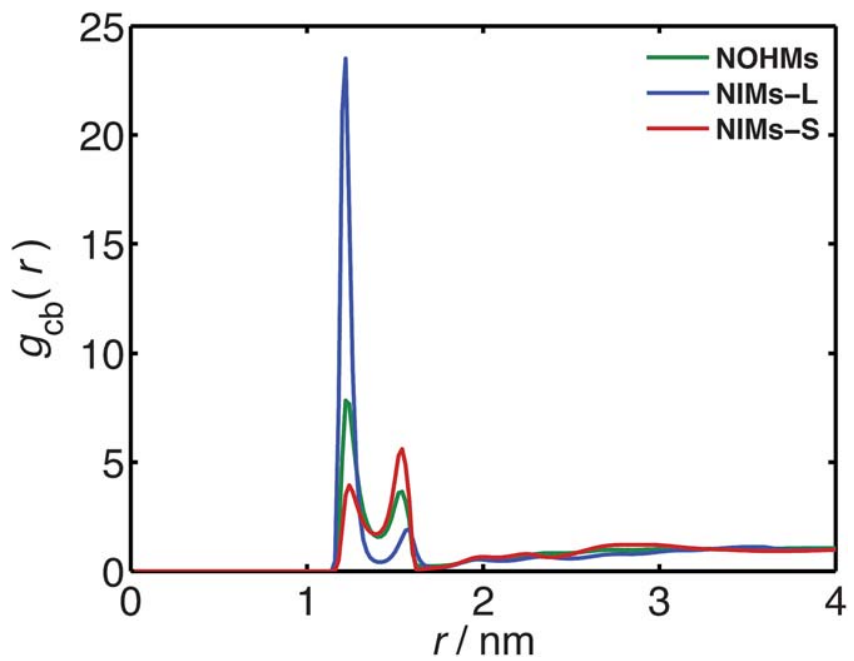

Fig. 6 Pair correlation functions between core particles and bead 2 for the base system $(N=$ 100). Colors are the same as for Fig. 5; the range of $r$ distances has been reduced with respect to Fig. 5 for clarity. 


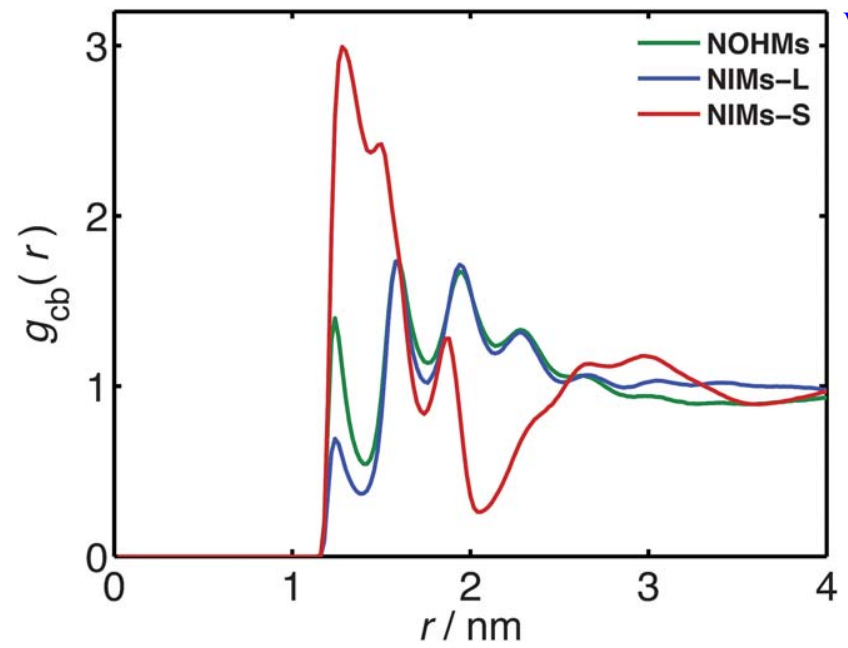

View Article Online

Fig. 7 Pair correlation functions between core particles and bead 7 for the base system $(N=100)$. Colors and $r$ scale are the same as for Fig. 6.

quite similar for the NIMs-L and NOHMs systems, being determined primarily by packing effects of the chains. The only noticeable difference is a lower first peak (on the core surface) for NIMs-L relative to NOHMs, due to the crowding effect of the charged beads seen in Fig. 6.

The pair correlation functions between positive and negative ions, $g_{\mathrm{pn}}$, are shown in Fig. 8. The functions are broadly similar, rising to a large value representing a contact ion pair at separation of $0.4 \mathrm{~nm}(=\sigma)$, but dropping quickly beyond this distance. The NIMs-S system has fewer counterions relative to the NIMS-L system at intermediate distances between 0.5 and $1 \mathrm{~nm}$, because of steric repulsions of the tertiary amines. However, the increased mobility of the tethered negative charge for NIMs-S, over the surface-immobilized charge for NIMs-L, leads to a higher first peak for the $g_{\mathrm{pn}}$, despite the increased steric hindrances from the chains.

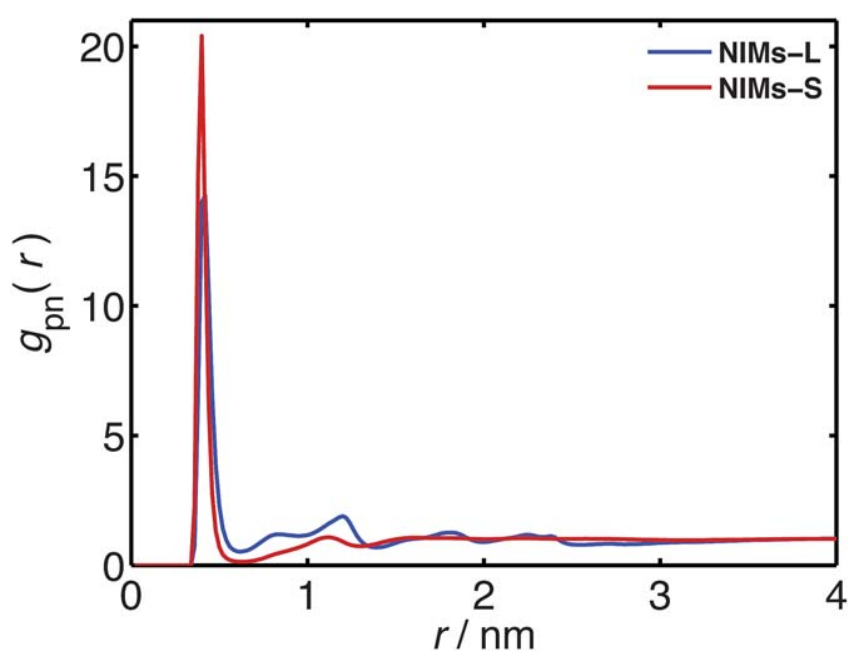

Fig. 8 Pair correlation functions between positive and negative ions for the base system $(N=100)$. Colors and $r$ scale are the same as for Fig. 6 . 
The correlation functions for the small system $(N=50)$ are close to throftide Online base system, with some variations of up to $20 \%$ in peak heights (and up to $40 \%$ for the high first peak of $g_{c c}$ for NIMs-L), but nearly identical peak features and positions. Some of these variations are due to the influence of the initial configuration, rather than system size, as confirmed by duplicate runs from different initial configurations. However, most of the dependence of $g_{c c}$ is due to the fact that even the $N=$ 100 system contains relatively few core particles. Larger systems, however, cannot currently be simulated for sufficiently long times to reach diffusive behavior.

\subsection{Dynamics}

A key dynamical feature that we have observed for our model NIMs systems is that, despite the strong electrostatic attractions between oppositely charged ions in the absence of a high-dielectric solvent, the chains have considerably mobility, initially on the surface of the core particles to which they are bound, and at later times between nearby cores. Movies showing the motion of chains and ions are provided as supplementary information to this article and illustrate this mobility quite clearly. $\dagger$ We quantify these chain motions by monitoring the fraction of chains, $f$, attached to their original surface sites or their original cores as a function of time (Fig. 9), calculated as described in Section 3. At time $t=0$, the fraction of positive ions attached to negative sites is very close to $100 \%$, reflecting the strong electrostatic interactions in the NIMs systems. The initial rapid drop-off of the fraction attached is likely a consequence of the assignment of any positive ion to a single negative surface site, even though the positive ions are frequently interacting strongly with multiple negative sites. A fraction of the cations shared between two such sites can get pulled from its original site within a short time. For later times, if we assume simple first-order kinetics for the detachment process, we anticipate that $\ln \left(f l f_{0}\right)=$ $-t / \tau$, where $\tau$ is a characteristic time and $f_{0}$ the initial fraction attached. As seen in Fig. 9, in which the logarithm of $f$ is plotted versus linear time, this assumption becomes reasonably good at longer times for ion detachments. For core detachments, first-order kinetics are approximately followed at all times. Characteristic times $\tau$ obtained from the inverse slopes of the curves are on the order of hundreds of ns for core detachments and tens of ns for ion detachments. A more quantitative

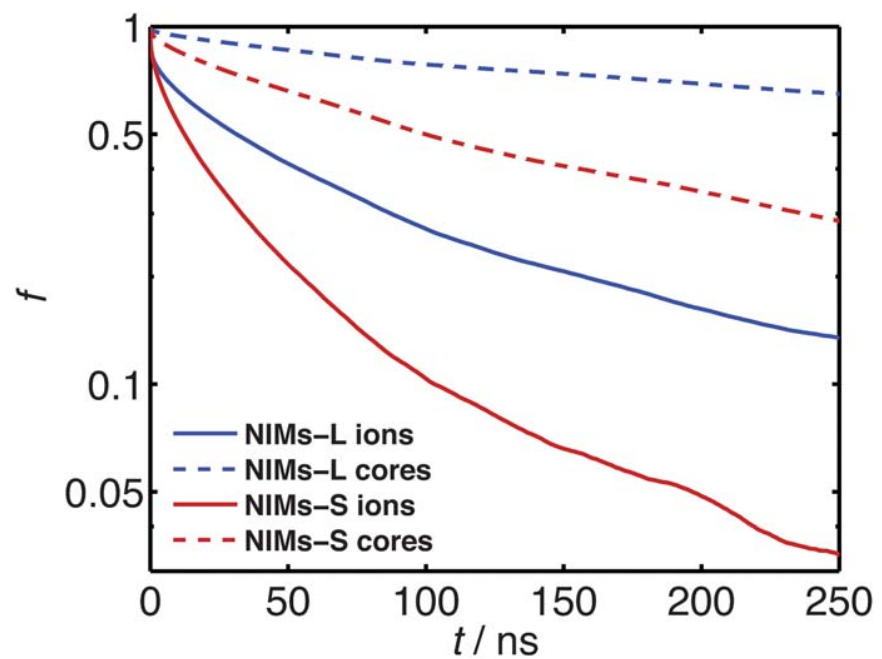

Fig. 9 Detachments versus time for the base NIMs systems $(N=100)$. Continuous lines are for positive ions detaching from negative ions and dashed lines for positive ions detaching from cores. Colors are as for Fig. 5. 
analysis is not warranted, given that first-order kinetics is only a rough appexoximate Online tion of the actual dynamics.

Detachment kinetics for chains in the NIMs-L system are slower than the NIMs-S system for both ions and cores. Most NIMs-L chains have their positively charged ions on the surface of the cores, with a smaller fraction one bead diameter from the cores (as evidenced from the height of the first and second peaks in Fig. 6). The NIMs-S chains do not have as many positive ions on the surface as NIMs-L chains, because of steric hindrances and the entropy loss of their arms when near the surface. ${ }^{31}$ They can become detached from the original ions and original cores more readily than the NIMs-L chains, as reflected in the more rapid decay of the corresponding curves in Fig. 9. Another reason for the enhanced mobility is the tethering of negative sites in the NIMs-S system. This allows chains to move more readily from one core to another, without a need for the two cores to come very near each other.

The mean-squared displacements (MSD) of core particles and centers of mass of chains are plotted in Fig. 10. For reference, the line of unit slope (corresponding to diffusive behavior) is also shown in the figure. Statistical noise is more pronounced at longer times. NOHMs particles reach diffusive behavior after approximately $300 \mathrm{~ns}$, when they have moved on average $\approx 1.5 \mathrm{~nm}$, a little less than a particle diameter away from their original positions. NIMs-L and NIMs-S core particles move more slowly than NOHMs particles, but reach near-diffusive behavior at comparable times. The estimated diffusion coefficients are $1.5 \pm 0.5 \times 10^{-8} \mathrm{~cm}^{2} \mathrm{~s}^{-1}$ for NOHMs, and $2 \pm 1 \times 10^{-9} \mathrm{~cm}^{2} \mathrm{~s}^{-1}$ for NIMs-L and NIMS-S cores.

We can obtain a rough approximation for the viscosity of these systems if we assume a hydrodynamic radius for the core particles along with their tethered or strongly held chains, and use the Stokes-Einstein equation to link diffusion coefficients of the core particles to the system viscosity. Using a hydrodynamic radius of $r_{h}=1.3 \mathrm{~nm}$ for both NIMs and NOHMs particles, we obtain a viscosity of $0.1 \mathrm{~Pa} \mathrm{~s}$ for our model NOHMs system and $1 \mathrm{~Pa} \mathrm{~s}$ for the model NIMs systems. We expect our coarse-grained models to have generally lower viscosities relative to their atomistic counterparts, because of the lack of torsional, bond bending and other orientational constraints. Experimentally measured NIMs viscosities ${ }^{1,3}$ at room temperatures are typically between 10 and $100 \mathrm{~Pa}$.

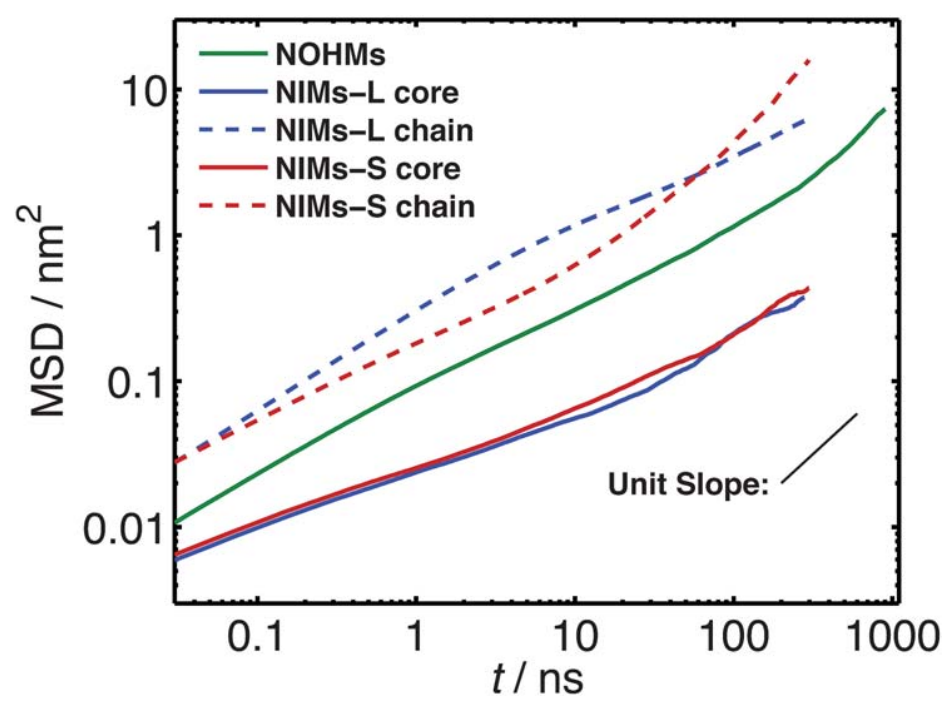

Fig. 10 Mean-squared displacements versus time. Dashed lines are for the chain centers of mass and continuous lines are for the core particles. Colors are as for Fig. 5. The short, thin black line represents a line of unit slope. 
In contrast to the behavior of the core particles, the motion of chairsefolawbe Online distinctly different behavior in the NIMs-S and NIMs-L systems. Chains travel faster than the core particles, but they do not display diffusive behavior until the cores themselves have become diffusive. This is a consequence of their motion primarily along the surfaces of the core particles until their detachment over time scales of hundreds of ns. The NIMs-S (star) chains move more slowly than NIMs$\mathrm{L}$ chains at intermediate times between 0.1 and $50 \mathrm{~ns}$. However, the NIMs-L linear chains are more strongly associated with the surface charges, and are slower in jumping from particle to particle, so their diffusion is eventually slowed down relative to their star counterparts. Diffusion coefficients of equal-sized uncharged linear and three-arm branched chains in solution ${ }^{32}$ are within $10 \%$ of each other for chain lengths from 8 to 100 . Thus, the main reason for the different diffusivities in our systems are electrostatic forces and differences in attachment intensities.

\section{Conclusions}

Two coarse-grained models for nanoscale ionic materials have been developed, consisting of core particles with attached charged sites and linear or three-arm branched chain counterions (NIMs-L and NIMs-S, respectively). The models are sufficiently simple so that their behavior can be simulated over sufficiently long ( $\mu \mathrm{s})$ time scales relevant for dynamics in these systems. A comparable model with tethered chains (NOHMs) has also been studied.

For the NIMs-L model of third-generation NIMs with primary amines, we find the amine groups preferentially on the surface of the cores, attracted to multiple negative sites on the same or different particles. As a result, core-core correlations for NIMs-L show a sharp first peak corresponding to two core particles with interpenetrating negative surface layers sharing contacts with a positive chain end. By contrast, the NIMs-S model of second-generation NIMs with secondary amines have significantly fewer positive groups near the core particle surface and do not show a contact peak in the core-core correlation function.

Despite the strong electrostatic forces that result in interaction energies at contact between positive groups on chains and core negative sites equal to $35 k_{\mathrm{B}} T$, chains are mobile. Over time scales of tens of ns, both linear and star chains detach from their original ions to nearby sites on the surface of a core particle. Over periods of hundreds of ns, chains move from one core particle to another, always keeping their positive sites in close contact with negative sites on the particles. Mobility of the NIMs-S tertiary chains is greater than of the NIMs-L linear chains. This is because tertiary chains have fewer of their positive ions on the core surfaces, because of steric hindrances and entropy loss, and also because tethering of the negative sites allows easier transfer from core to core.

Nanoparticles in both NIMs and NOHMs systems become diffusive after approximately $300 \mathrm{~ns}$. Diffusivity of the cores is greater by a factor of ten in the NOHMs system relative to NIMs, also resulting in a lower estimated viscosity. Chains in NIMs-L and NIMs-S systems show distinct dynamical behavior. Linear chains move faster on the surface of the cores than the bulkier star chains at intermediate times up to $50 \mathrm{~ns}$, but are more strongly associated with their original cores and thus slower at longer times.

Our findings on chain mobility are in agreement with a recent experimental study of canopy dynamics on NIMs, ${ }^{15}$ that concluded that "the liquid-like behavior in NIMs is due to rapid exchange of the [canopy chains] between the ionically modified nanoparticles." Chain hopping between surface sites and from core particle to core particle provide a mechanism for cation-based superionic conductivity (that is, higher than anticipated from their fluidity), as hypothesized in ref. 1. These observations also provide the basis for understanding why replacement of small counterions with large organic ones turns NIMs from high-melting solids into liquids. ${ }^{1}$ 
Only a single set of model parameters were examined in this study. In thew futurefe Online we plan to investigate the influence of core particle size and volume fraction, chain composition and length, and temperature, on the structure, fluidity, and conductivity of these systems. More quantitative comparisons to specific experimental systems should also be possible.

\section{Acknowledgements}

This paper is based on work supported by Award KUS-C1-018-02 made by King Abdullah University of Science and Technology (KAUST) and by grants DE-SC0002128 from the US Department of Energy, Office of Basic Energy Sciences and CBET-1033155 from NSF. Simulations were performed on the Della cluster of PICScIE, a facility supported by Princeton University. The authors would like to thank Prof. Fernando Escobedo for suggesting the NOHMs model, and Prof. Emmanuel Giannelis and Lynden Archer for many helpful discussions.

\section{References}

1 A. B. Bourlinos, K. Raman, R. Herrera, Q. Zhang, L. A. Archer and E. P. Giannelis, J. Am. Chem. Soc., 2004, 126, 15358-15359.

2 A. B. Bourlinos, R. Herrera, N. Chalkias, D. D. Jiang, Q. Zhang, L. A. Archer and E. P. Giannelis, Adv. Mater., 2005, 17, 234-236.

3 A. B. Bourlinos, S. R. Chowdhury, R. Herrera, D. D. Jiang, Q. Zhang, L. A. Archer and E. P. Giannelis, Adv. Funct. Mater., 2005, 15, 1285-1290.

4 A. B. Bourlinos, A. Stassinopoulos, D. Anglos, R. Herrera, S. H. Anastasiadis, D. Petridis and E. P. Giannelis, Small, 2006, 2, 513-516.

5 Q. Li, L. J. Dong, J. F. Fang and C. X. Xiong, ACS Nano, 2010, 4, 5797-5806.

6 N. Fernandes, P. Dallas, R. Rodriguez, A. B. Bourlinos, V. Georgakilas and E. P. Giannelis, Nanoscale, 2010, 2, 1653-6.

7 A. B. Bourlinos, E. P. Giannelis, Q. Zhang, L. A. Archer, G. Floudas and G. Fytas, Eur. Phys. J. E, 2006, 20, 109-117.

8 J. F. Brennecke and E. J. Maginn, AIChE J., 2001, 47, 2384-2389.

9 P. Agarwal, H. B. Qi and L. A. Archer, Nano Lett., 2010, 10, 111-115.

10 R. Rodriguez, R. Herrera, L. A. Archer and E. P. Giannelis, Adv. Mater., 2008, 20, 4353-4358.

11 R. Rodriguez, R. Herrera, A. B. Bourlinos, R. P. Li, A. Amassian, L. A. Archer and E. P. Giannelis, Appl. Organomet. Chem., 2010, 24, 581-589.

12 Q. Zhang and L. A. Archer, Langmuir, 2002, 18, 10435-10442.

13 J. L. Nugent, S. S. Moganty and L. A. Archer, Adv. Mater., 2010, 22, 3677-80.

14 D. Kim and L. A. Archer, Langmuir, 2011, 27, 3083-3094.

15 M. L. Jespersen, P. A. Mirau, E. von Meerwall, R. A. Vaia, R. Rodriguez and E. P. Giannelis, ACS Nano, 2010, 4, 3735-3742.

16 J. M. Oommen, M. M. Hussain, A. H. M. Emwas, P. Agarwal and L. A. Archer, Electrochem. Solid-State Lett., 2010, 13, K87-K88.

17 T. I. Morrow and E. J. Maginn, J. Phys. Chem. B, 2002, 106, 12807-12813.

18 T. Y. Yan, C. J. Burnham, M. G. Del Popolo and G. A. Voth, J. Phys. Chem. B, 2004, 108, $11877-11881$.

19 E. J. Maginn, J. Phys.: Condens. Matter, 2009, 21, 373101.

20 G. D. Smith, D. Bedrov, L. W. Li and O. Byutner, J. Chem. Phys., 2002, 117, 9478-9489.

21 F. W. Starr, J. F. Douglas and S. C. Glotzer, J. Chem. Phys., 2003, 119, 1777-1788.

22 V. Pryamtisyn, V. Ganesan, A. Z. Panagiotopoulos, H. J. Liu and S. K. Kumar, J. Chem. Phys., 2009, 131, 221102.

23 H. Y. Yu and D. L. Koch, Langmuir, 2010, 26, 16801-16811.

24 L. Constantinou and R. Gani, AIChE J., 1994, 40, 1697-1710.

25 B. Smit, J. Chem. Phys., 1992, 96, 8639-8640.

26 J. D. Weeks, D. Chandler and H. C. Andersen, J. Chem. Phys., 1971, 54, 5237-47.

27 J. S. Smith, D. Bedrov and G. D. Smith, Compos. Sci. Technol., 2003, 63, 1599-1605.

28 A. M. Awwad, A. H. Al-Dujaili and H. E. Salman, J. Chem. Eng. Data, 2002, 47, 421-424.

29 P. R. Gray, P. J. Hurst, S. H. Lewis and R. G. Meyer, Analysis and Design of Analog Integrated Circuits, p. 40, Wiley (New York), 5th edn, 2009.

30 S. Plimpton, J. Comput. Phys., 1995, 117, 1-19.

31 A. Chremos, P. J. Camp, E. Glynos and V. Koutsos, Soft Matter, 2010, 6, 1483-1493.

32 C. L. Fu, W. Ouyang, Z. Y. Sun and L. An, J. Chem. Phys., 2007, 127, 044903. 